\title{
DESI-MS as a tool for direct lipid analysis in cultured cells
}

\author{
Anna Bodzon-Kulakowska • Tomasz Cichon • \\ Agnieszka Golec • Anna Drabik · Joanna Ner • \\ Piotr Suder
}

Received: 25 September 2013/ Accepted: 19 April 2014/Published online: 7 May 2014

(C) The Author(s) 2014. This article is published with open access at Springerlink.com

\begin{abstract}
Desorption electrospray ionization may be used as a fast and convenient method for analysis and identification of lipids in the cell culture. Oxidative stress, which usually involves changes in lipids, was used as a model of pathology to show the utility of this analysis methodology. This paper addresses the surface preparation of cell culture slides, induction of oxidative stress, and cell monolayer culture preparation as well as optimization of the analysis. Advantages and drawbacks of the method were also discussed.
\end{abstract}

Keywords Desorption electrospray ionization (DESI) - Cell culture $\cdot$ Lipid analysis $\cdot$ Oxidative stress

\section{Introduction}

Desorption electrospray ionization (DESI) is a mass spectrometry imaging (MSI) method used in the biological sciences for surface analysis. An electrospray emitter is used to generate charged microdroplets which are directed at the surface for analysis, where they create a thin liquid film which dissolves the

A. Bodzon-Kulakowska - T. Cichon .

A. Golec · A. Drabik · J. Ner · P. Suder $(\bowtie)$

AGH University of Science and Technology, Kraków,

Poland

e-mail: piotr.suder@agh.edu.pl analyte(s). Secondary microdroplets containing the analyte are produced by the impact of subsequent primary droplets. The analyte is introduced into the MS inlet where the ions are analysed by mass spectrometer (Girod et al. 2010a; Takáts et al. 2004) (Fig. 1).

Compounds below 2,000 Da (such as lipids) can be measured and their distribution on the surface visualized, while particular molecules may be identified using MS/MS fragmentation. Additional advantages are that DESI does not require laborious sample preparation and can be performed at ambient conditions. Its spatial resolution reaches a pixel size of ca. $50 \times 50 \mu \mathrm{m}$ or better (down to $10 \times 10 \mu \mathrm{m}$ ) with modification of the DESI source (Laskin et al. 2012). Taken together, DESI is a fast and convenient tool for lipid analysis in biological samples (Eberlin et al. 2011).

Oxidative stress is a pathological state of the cell/ organism where the amount of oxygen radicals increases but cannot be counterbalanced by the internal antioxidant defense system, or when the level of oxidative radicals remains at a physiological state, but the defense system is impaired. This may lead to the oxidative damage of proteins, lipids, and DNA, which usually leads to apoptosis (Feeney et al. 2008). Lipids are especially sensitive to this pathophysiology and undergo peroxidation, which results in a number of highly-reactive species that can modify other proteins and lipids, yielding aldehydes and polymerized carbonyl compounds (Weismann and Binder 


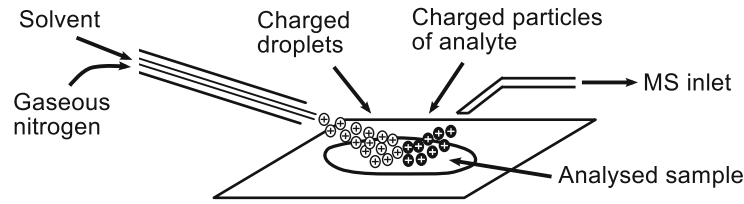

Fig. 1 DESI ion source

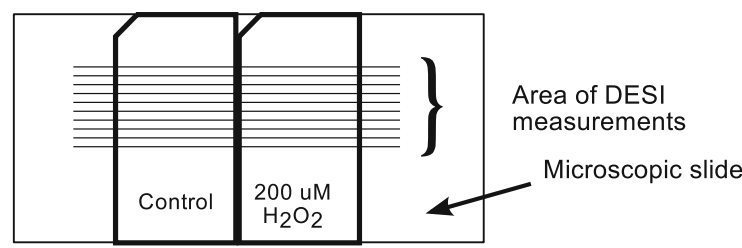

Fig. 2 DESI measurements-arrangement of the glasses with the monolayer cell culture on the DESI holder

2012). As lipid alterations are involved in oxidative stress, the model in this study was chosen to show the usefulness of DESI lipid analysis in cell culture.

\section{Materials and methods}

If not otherwise stated, all chemicals were purchased from Sigma-Aldrich (Poznan, Poland) and were of the highest purity available.

\section{Surface preparation for cell culture}

The DESI ion source (OMNIspray, Prosolia, Indianapolis, IN, USA) was equipped with a holder for standard microscopic glass slides $(75 \times 25 \mathrm{~mm})$ as a base for surface scanning. To combine cell monolayers from various experiments on a single holder, cells were cultured on glass slides $(15 \times 25 \mathrm{~mm})$ and individual glasses were combined on a DESI holder (Fig. 2). The upper-left corner of each glass was cut at the angle of $45^{\circ}$ to allow for immediate identification of the upper surface of the glass slide.

Before cell culturing, glass slides were washed with hexane for $5 \mathrm{~min}$ in an ultrasonic bath (SonorexDigitec, Bandelin-Electronic, Berlin, Germany) and then with $95 \%$ ethanol for $5 \mathrm{~min}$. The remaining work was done in a laminar airflow cabinet (NU-480, NuAire Inc., Plymouth, MN, USA) to ensure aseptic conditions. The ethanol was evaporated, slides washed twice with sterile water and covered with a $0.02 \mathrm{mg} / \mathrm{ml}$ solution of poly-L-lysine (30-70 kDa; P2636, SigmaAldrich) overnight. Immediately before cell culturing, the slides were removed from the poly-L-lysine and washed twice with PBS.

Cell culture

A fibroblast cell line (Balb/3T3 cells, Ludwik Hirszfeld Institute of Immunology and Experimental Therapy, Polish Academy of Sciences, Wroclaw, Poland, stored in a liquid nitrogen) was thawed at $37{ }^{\circ} \mathrm{C}$. The cell suspension was centrifuged at $300 \times g$ for $7 \mathrm{~min}$, the cryoprotectant medium removed and the cells were resuspended in Eagle's Minimal Essential Medium (M2279, Sigma-Aldrich) supplemented with $10 \% \mathrm{v} / \mathrm{v}$ Foetal Bovine Serum (F9665, Sigma-Aldrich), $1 \%$ v/v antibiotic antimycotic solution (A5955, SigmaAldrich) and $1 \mathrm{mM}$ glutamine (49419, SigmaAldrich). The cells were centrifuged at $300 \times g$ for $4 \mathrm{~min}$, resuspended in cell culture medium, seeded on the poly-L-lysine coated slides and cultured at $5 \%$ $\mathrm{CO}_{2}, 37{ }^{\circ} \mathrm{C}$ and $95 \%$ relative humidity (DH5810E, NuAire Inc.) for 6 days until confluent.

\section{Oxidative stress}

The simplest method to induce oxidative stress in cell culture was to disturb the prooxidant-antioxidant balance by increasing radical load, which can be accomplished by adding hydrogen peroxide (or other agents) to the cell culture medium (Gille and Joenje 2002). Glass slides with confluent cell monolayers were removed from the Petri dish and placed in a new container with a new portion of medium (control) or medium supplemented with $\mathrm{H}_{2} \mathrm{O}_{2}(200 \mu \mathrm{M})$ for $1 \mathrm{~h}$. One set of glass slides was used for DESI analysis and the other for analysis of cell viability by trypan blue staining (Patterson 1979).

Cell culture preparation for DESI analysis

Immediately before DESI, the medium was removed from the Petri dish containing the glass slide with the cell monolayer. To remove salts and other remainings of the cell culture medium, the slide was rinsed twice with a volume of warm $\left(37^{\circ} \mathrm{C}\right) 150 \mathrm{mM}$ ammonium 
acetate buffer, pH 7.1 (A7330, Sigma-Aldrich) for $5 \mathrm{~s}$. The glass slide was removed from the dish, dried using a stream of dry nitrogen directed at the surface of the cell monolayer and frozen at $-80{ }^{\circ} \mathrm{C}$ until DESI analysis. The isotonic ammonium acetate solution was volatile enough to evaporate quickly (Piwowar et al. 2013).

\section{DESI analysis}

Glass slides with control and hydrogen peroxidetreated cell monolayers were placed into the DESI holder (Fig. 2). During the imaging experiments, cell monolayers were scanned using a 2D moving stage in horizontal rows separated by a $0.2 \mathrm{~mm}$ distance, and 50 rows were measured at $100 \mu \mathrm{m} / \mathrm{s}$ with a single mass spectrum saved every $1.5 \mathrm{~s}$ (spatial resolution of ca. $170 \mathrm{dpi})$. A methanol : water solution $(1: 1 \mathrm{v} / \mathrm{v})$ containing $1 \mu \mathrm{M}$ surfactin was sprayed at a constant flow rate of $2.0 \mu \mathrm{l} / \mathrm{min}$. The mixture of water and methanol is a standard solution used for DESI analyses and the addition of surfactin enhanced signal quality, especially in the negative ion mode. Control and $200 \mu \mathrm{M} \mathrm{H}_{2} \mathrm{O}_{2}$-treated cells were measured during a single analysis (Fig. 2), and Data Analysis ver 4.0 software (Bruker-Daltonics, Bremen, Germany) was used for spectral analysis, while the BioMap freeware (http://www.maldi-msi.org) (Novartis, Basel, Switzerland) was used for image generation.

An DESI OMNIspray ion source combined with an AmaZon ETD MS (Bruker-Daltonics) was operated under the HyStar ver. 3.2 software supervision (Bruker Daltonics). HyStar coordinated work of the Omnispray 2D software (Prosolia) controlling the DESI stage movements, and the Bruker's TrapControl ver. 7.0 software (Bruker Daltonics) controlling mass spectrometer activity. Mass spectrometer settings were as follows: scan range $300-950 \mathrm{~m} / \mathrm{z}$, capillary voltage of $+4,200 \mathrm{~V}$, ion trap operating in the ICC mode set to 300,000 ions/cycle, maximal ion accumulation time of $200 \mathrm{~ms}$ and a heated capillary temperature of $220^{\circ} \mathrm{C}$. The negative ion mode was selected, as it yielded more information during lipid analysis than positive polarization. The most abundant peaks from the cell culture sample were further identified using MS/MS fragmentation (collision induced dissociation energy $1.2 \mathrm{~V}$, precursor isolation width 4.0 Da).
Statistics

Eighty scans were taken to obtain accumulated spectra for every single line from the analysed samples (control and $200 \mu \mathrm{M} \mathrm{H}_{2} \mathrm{O}_{2}$ ). The intensities of each chosen peak were averaged, and the standard deviations and $p$ values were calculated using the Student's $t$ test.

\section{Results}

Oxidative stress and cell viability

After $1 \mathrm{~h}$ of incubation in the appropriate media, a set of glass slides was subjected to viability test using trypan blue staining. In the control sample and cells subjected to oxidative stress, the viability of the cells was unchanged. However, cells subjected to the $200 \mu \mathrm{M}$ of $\mathrm{H}_{2} \mathrm{O}_{2}$ started to show morphological signs of oxidative stress by changing their irregular flattened, extended shape and rounding (Kiyoshima et al. 2012).

\section{DESI analysis}

To obtain average spectra for each sample (control and $200 \mu \mathrm{M} \mathrm{H}_{2} \mathrm{O}_{2}$ ), 80 mass spectra were accumulated for every surface (Figs. 2 and 3). From the accumulated spectra, ions of interest were selected, and the peaks corresponding to particular lipids, as well as those expected to originate from the background, were considered.

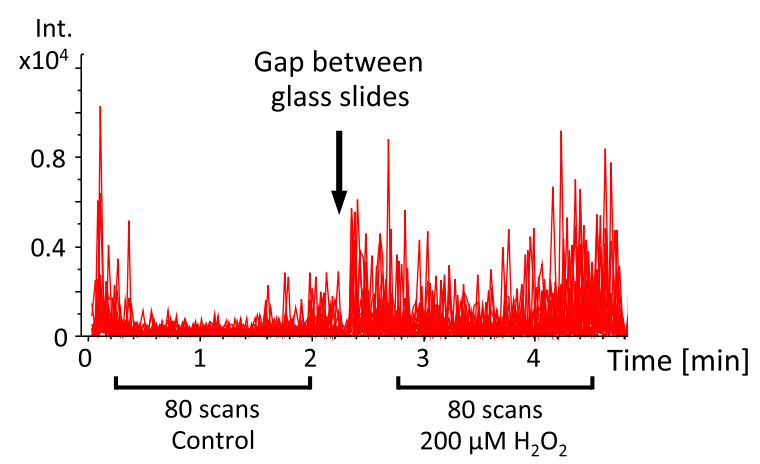

Fig. 3 Selection of the mass spectra (scans) for averaging. The plotted lines represent a chromatogram of extracted ion at the $885.5 \mathrm{~m} / \mathrm{z}$ peak, characteristic for the areas covered by cells. Arrow shows the gap between the glass slides with control cells and cells treated with $200 \mu \mathrm{M} \mathrm{H}_{2} \mathrm{O}_{2}$ 


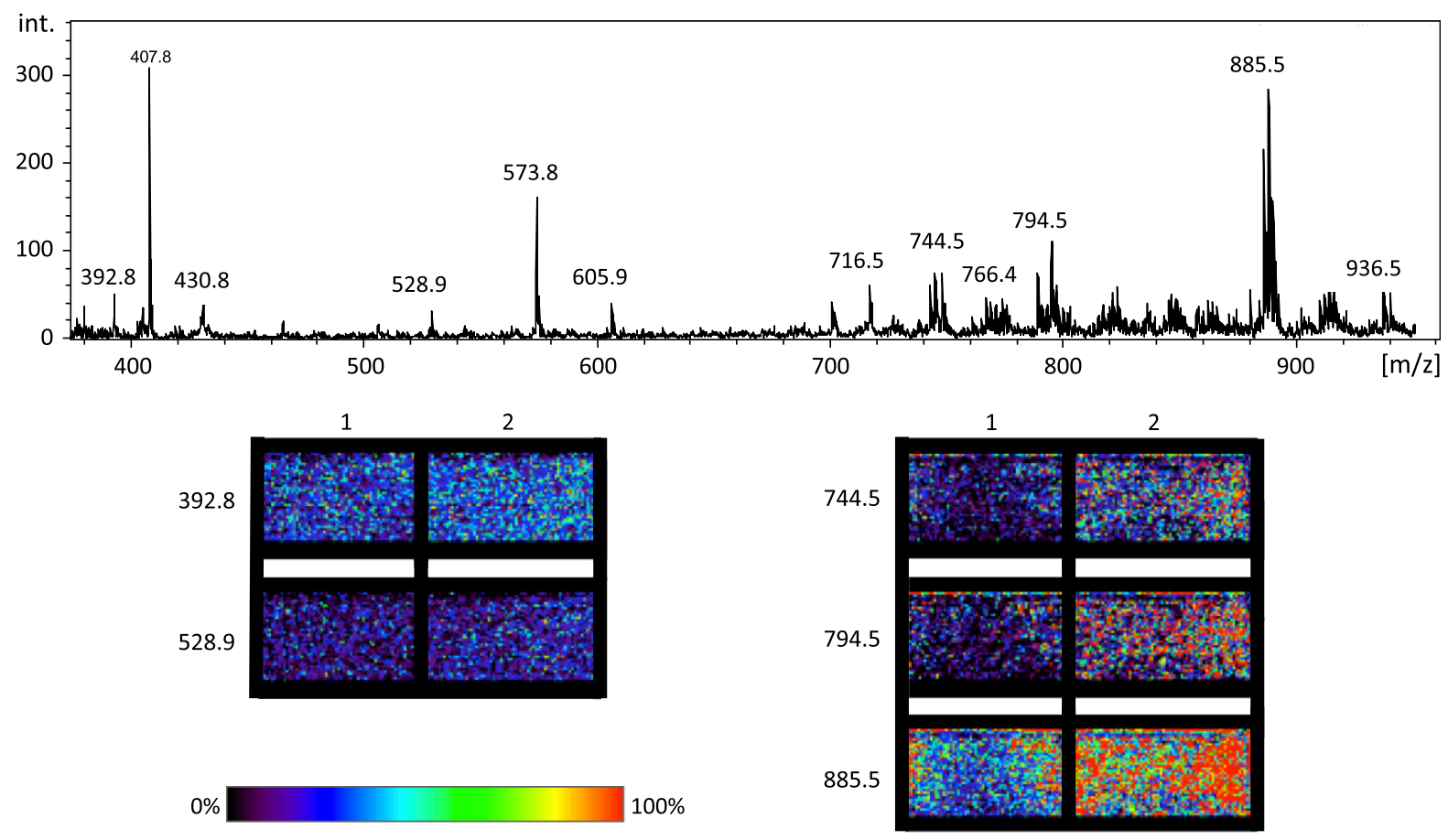

Fig. 4 Average spectrum from the cell culture treated with $200 \mu \mathrm{M}$ of hydrogen peroxide. Below the spectrum are images from Biomap indicating the abundances of the selected peaks in every sample $\left(1\right.$ control, $\left.2200 \mu \mathrm{M} \mathrm{H}_{2} \mathrm{O}_{2}\right)$

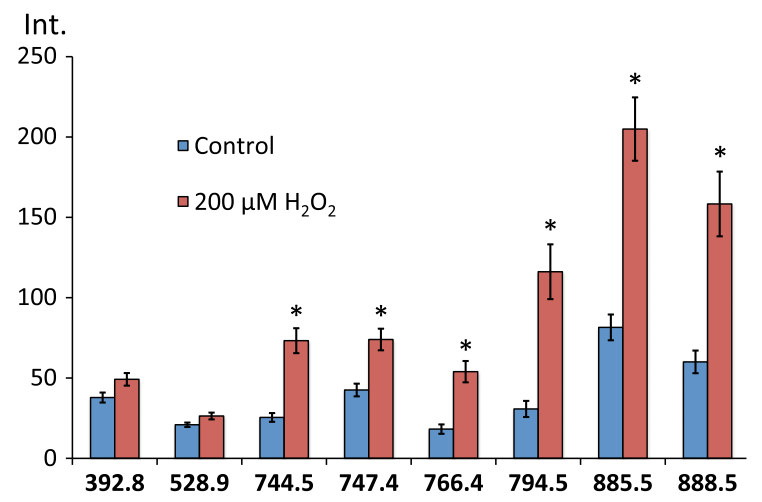

Fig. 5 Fluctuation of selected ion intensities after treatment with hydrogen peroxide. Peaks at 392.8 and 528.9 were background, and indicated insignificant variability among samples, in contrast to those representing lipids, shown on the graph. Differences between corresponding values, for the same peaks, marked with an asterisk are significant ( $p$ value $<0.05$ )

Figure 4 shows the spectrum from cells treated with $200 \mu \mathrm{M}$ of hydrogen peroxide, averaged from the 80 spectra. The peaks corresponding to particular lipids were selected for analysis, as well as some peaks from the background (for the comparison purpose-as a negative control).
Table 1 Characteristic group loss from sn-3 position for lipids easily detected during MS/MS analyses

\begin{tabular}{|c|c|c|}
\hline Lipid name & Abbreviation & $\begin{array}{l}\text { Group loss } \\
\text { from sn-3 } \\
\text { position }\end{array}$ \\
\hline Glycerophosphocholine & GPCho (PC) & $\begin{array}{c}{[\mathrm{M}-\mathrm{H}-15]^{-},} \\
{[\mathrm{M}-\mathrm{H}-} \\
60]^{-},[\mathrm{M}- \\
\mathrm{H}-84]^{-}\end{array}$ \\
\hline Glycerophosphoethanolamine & GPEtn (PE) & {$[\mathrm{M}-\mathrm{H}-104]^{-}$} \\
\hline Glycerophosphoglycerol & GPGro (PG) & {$[\mathrm{M}-\mathrm{H}-74]^{-}$} \\
\hline Glycerophosphoinositol & GPIno (PI) & {$[\mathrm{M}-\mathrm{H}-162]^{-}$} \\
\hline Glycerophosphoserine & GPSer (PS) & {$[\mathrm{M}-\mathrm{H}-87]^{-}$} \\
\hline Plasmanylphospholipid & Plasm-PE & {$[\mathrm{M}-\mathrm{H}-43]^{-}$} \\
\hline Sulfatide & ST & {$[\mathrm{M}-\mathrm{H}-18]^{-}$} \\
\hline
\end{tabular}

Changes in peak intensities after hydrogen peroxide administration are shown in Fig. 5.

MS/MS fragmentation

DESI allowed identification of analysed compounds using MS/MS fragmentation. In the negative ion 
Table 2 Masses for pseudomolecular ions and neutrals characteristic for different fatty acyl substituents

\begin{tabular}{llll}
\hline & Carboxylic acid $[\mathrm{M}-\mathrm{H}]^{-}$ & Carboxylic acid & Ketene \\
\hline$(14: 0)$ & 227 & 228 & 210 \\
$(16: 1)$ & 253 & 254 & 236 \\
$(16: 0)$ & 255 & 256 & 238 \\
$(18: 2)$ & 279 & 280 & 262 \\
$(18: 1)$ & 281 & 282 & 264 \\
$(18: 0)$ & 283 & 284 & 266 \\
$(20: 4)$ & 303 & 304 & 286 \\
$(20: 3)$ & 305 & 306 & 288 \\
$(20: 2)$ & 307 & 308 & 290 \\
$(22: 6)$ & 327 & 328 & 310 \\
$(24: 1)$ & 365 & 366 & 348 \\
\hline
\end{tabular}

mode, identification of lipids is not a very demanding task. Usually these compounds form pseudomolecular ions $[\mathrm{M}-\mathrm{H}]^{-}$, which fragment by losing a characteristic portion attached to the sn-3 position of the glycerol backbone (Table 1). Characteristic peaks from such fragmentation may be present on the spectrum.

The MS/MS spectra of lipids showed losses of two different neutrals, carboxylic acid [M-H$\left.\mathrm{R}_{1,2} \mathrm{CH}_{2} \mathrm{COOH}\right]$ and the corresponding ketene [M$\left.\mathrm{H}-\mathrm{R}_{1,2} \mathrm{CH}=\mathrm{C}=\mathrm{O}\right]^{-}$. Examples of masses for pseudomolecular ions and neutrals characteristic for different fatty acyl substituents are listed in the Table 2. Lipids were identified according to Tables 1 and 2 in addition to data from the literature (Manicke et al. 2008; Hsu and Turk 2009, 2005).

Moreover, neutral loss for the sn-2 position was more favourable, giving rise to more intense peaks, and the position of the two carboxylic acids may be distinguished. Excellent reviews on this topic may be found in Hsu and Turk (2009) and Girod et al. (2010b). An example of MS/MS spectrum for the ion at $788.5 \mathrm{~m} / \mathrm{z}$ is shown in Fig. 6. The main peak on the MS/MS spectrum at $701.4 \mathrm{~m} / \mathrm{z}$ resulted from serine loss $(788-701=87)$, the peaks at 419.0 and 437.0 resulted from neutral loss of $18: 1$ as a carboxylic acid $(788.5-87-419=282.5)$ and ketene $(788.5-87-437=264.5)$ as well as a small peak at $417 \mathrm{~m} / \mathrm{z}$, which was from neutral loss of 18:0 (788.5 - 87 - $417=284.5)$ (see Table 2). These data indicated the ion at $788.5 \mathrm{~m} / \mathrm{z}$ was glycerophosphoserine (18:0/18:1).

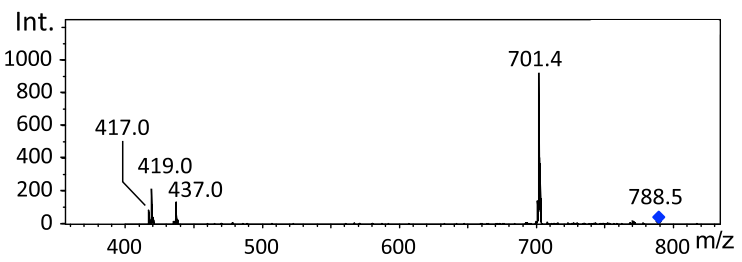

Fig. $6 \mathrm{MS} / \mathrm{MS}$ fragmentation of the ion at $788.5 \mathrm{~m} / \mathrm{z}$. See the text for details

Table 3 MS/MS identification of several lipids whose level changed due to oxidative stress

\begin{tabular}{ll}
\hline 744.5 & Glycerophosphoethanolamine PE (18:0/18:1) \\
747.4 & Glycerophosphoglycerol PG (18:0/18:1) \\
766.4 & Glycerophosphoethanolamine PE (18:0/20:4) \\
885.5 & Glycerophosphoinositol PI (18:0/20:4) \\
888.5 & Sulfatide ST (18:1/24:1) \\
\hline
\end{tabular}

Some of the peaks from the cell culture which changed due to oxidative stress were identified (Table 3).

\section{Discussion}

DESI offered a rapid and convenient way of sample measurement/imaging compared to other mass spectrometry imaging techniques based on MALDI and SIMS ion sources that require high vacuum inside the source. The entire procedure for preparing the single glass slide and adherent cell monolayer culture required ca. $30 \mathrm{~s}$ with an additional $10 \mathrm{~min}$ for drying. DESI construction on the 2D moving stage allowed for placement of glass/plastic slides with adherent cells from various experiments side by side, which allowed the measurements to be performed simultaneously. This was crucial for comparative research between cells treated with various substances or cells with different pathologies.

As with most analytical techniques, DESI was not without drawbacks. The main disadvantage was the limited range of $\mathrm{m} / \mathrm{z}$ that could be analysed. Compounds below 2,000 Da were easily analysed, but for higher masses, a more sophisticated optimization of the system, as well as prescanning of the surface might be necessary. DESI allowed imaging of the surface, but for cell culture analyses, the resolution of the 
system did not allow for detailed analyses of a single cell. This limitation was due to two variables: (1) the smallest available spraying surface for acquisition of a single pixel, and (2) the analysis time. The DESI interface was suitable for imaging macroscopic objects, such as brain structures (Eberlin et al. 2011). Recent publications about DESI-related analyses indicate that resolutions better than ca. 2,000 dpi are not currently available at the present stage of ion source development (Laskin et al. 2012).

Analysis time was connected to image resolution, and higher resolution required the system to acquire more scans, which served as points (pixels) of the image. Thus, twofold better resolution required fourfold more time to complete the analysis. Another disadvantage of DESI analysis was related to proper optimization of the DESI source. There were several parameters crucial for obtaining a quality spectrum and small changes in source geometry (nebuliser capillary angle, its distance to the surface and distance to the MS inlet), as well as other settings such as nebulising gas pressure, solvent flow and capillary voltage, may affect the analyses. Optimisation based on artificial, easilyionisable standards (e.g. rhodamine or bradykinin) may be insufficient for more complex samples such as cells cultured on glass (Bodzon-Kulakowska et al. 2014). Thus, additional sets of samples only subjected to ion source optimisation should be prepared. Another thing is that the analysed surface should be as even as possible, because small changes in the distance between nebuliser capillary and the surface may influence peak intensity.

The effects of oxidants may be easily detected in cell monolayers with the aid of DESI-equipped mass spectrometers. Cell cultures did not require advanced preparation methods or any special type of visualization of the changes occurring between samples prior to the MS analysis. With respect to analysis of cellular membrane lipid in various cell cultures, DESI appeared to be the most rapid, convenient, reliable, and sensitive method of analysis. In direct contrast to staining techniques, radioimmunoassays, lipid derivatization or homogenate analyses, DESI did not introduce any additional substances to the sample, and advanced, multi-step procedures were not necessary. Changes in abundances of ions corresponding to lipids under oxidative stress, could be seen. Additionally, using DESI in combination with MS/MS analysis allowed these species to be identified.
Categorical judgements about the biological significance of the effects observed were not possible based on these preliminary experiments, as this work was devoted to evaluating the application of DESI as a tool to analyse cell changes. However, the effects may be connected to several processes in the cells. With the aid of DESI, a direct destructive effect of hydrogen peroxide on the cell membranes of the living cells could be observed. Interactions between reactive oxygen species and the cell membrane could cause partial destruction which would expose lipids, rendering them more susceptible to ionization. This may be supported by the presence of greater numbers of dead cells (trypan blue) after introduction of the higher amount of $\mathrm{H}_{2} \mathrm{O}_{2}$ into the cell cultures. Another explanation could be due to a more subtle effect, based on the interaction between the intracellular signalling system and extracellular reactive oxygen species. In this case, cellular reactivity may have caused changes in cell membranes which resulted in increased exposition of lipid molecules working as a shield against an adverse extracellular environment.

The data could not show the formation of aldehydes and polymerized carbonyl compounds (mentioned by other authors) that occurred during oxidative stress in tissues or cells. Lack of observation of those forms may be connected to the too short interaction time of oxidative species with the cells. Alternatively, these species may be undetectable due to their minor concentration in the investigated material, and optimization of the MS system for particular ions or application of the MRM (multiple reaction monitoring) procedure would allow for successful identification of such modified lipids.

Despite the fact that these results are preliminary, the problem of oxidative stress in the cell culture demands further studies. Our data showed the utility of this type of mass spectrometry imaging for fast analysis of lipids from the cell culture.

Acknowledgments The research was supported by the Foundation for Polish Science-POMOST Programme (POMOST/2011-3/1) co-financed by the European Union within the European Regional Development Fund, The Polish National Science Center 3744/B/H03/2011/40, and EuroNanoMed "META" 05/EuroNanoMed/2012.

Open Access This article is distributed under the terms of the Creative Commons Attribution License which permits any use, distribution, and reproduction in any medium, provided the original author(s) and the source are credited. 


\section{References}

Bodzon-Kulakowska A, Drabik A, Ner J, Kotlinska JH, Suder P (2014) Desorption electrospray ionisation (DESI) for beginners-how to adjust settings for tissue imaging. Rapid Commun Mass Spectrom 28:1-9

Eberlin LS, Ferreira CR, Dill AL, Ifa DR, Cooks RG (2011) Desorption electrospray ionization mass spectrometry for lipid characterization and biological tissue imaging. Biochim Biophys Acta 1811:946-960

Feeney CJ, Frantseva MV, Carlen PL, Pennefather PS, Shulyakova N, Shniffer C, Mills LR (2008) Vulnerability of glial cells to hydrogen peroxide in cultured hippocampal slices. Brain Res 1198:1-15

Gille JJP, Joenje H (2002) Cell culture models for oxidative stress: superoxide and hydrogen peroxide versus normobaric hyperoxia. Mutat Res 275:405-414

Girod M, Cheng J, Cooks R, Shi Y (2010a) Mapping lipid alterations in traumatically injured rat spinal cord by desorption electrospray ionization imaging mass spectrometry. Anal Chem 83:207-215

Girod M, Shi Y, Cheng J-X, Cooks RG (2010b) Desorption electrospray ionization imaging mass spectrometry of lipids in rat spinal cord. J Am Soc Mass Spectrom 21: 1177-1189

Hsu F-F, Turk J (2005) Studies on phosphatidylserine by tandem quadrupole and multiple stage quadrupole ion-trap mass spectrometry with electrospray ionization: structural characterization and the fragmentation processes. J Am Soc Mass Spectrom 16:1510-1522
Hsu F-F, Turk J (2009) Electrospray ionization with low-energy collisionally activated dissociation tandem mass spectrometry of glycerophospholipids: mechanisms of fragmentation and structural characterization. J Chromatogr B 877:2673-2695

Kiyoshima T, Enoki N, Kobayashi I, Sakai T, Nagata K, Wada H, Fujiwara H, Ookuma Y, Sakai H (2012) Oxidative stress caused by a low concentration of hydrogen peroxide induces senescence-like changes in mouse gingival fibroblasts. Int J Mol Med 30:1007-1012

Laskin J, Heath BS, Roach PJ, Cazares L, Semmes OJ (2012) Tissue imaging using nanospray desorption electrospray ionization mass spectrometry. Anal Chem 84:141-148

Manicke NE, Wiseman JM, Ifa DR, Cooks RG (2008) Desorption electrospray ionization (DESI) mass spectrometry and tandem mass spectrometry (MS/MS) of phospholipids and sphingolipids: ionization, adduct formation, and fragmentation. J Am Soc Mass Spectrom 19:531-543

Patterson MK Jr (1979) Measurement of growth and viability of cells in culture. Methods Enzymol 58:141-152

Piwowar AM, Keskin S, Delgado MO, Shen K, Hue JJ, Lanekoff I, Ewing AG, Winograd N (2013) C60-ToF SIMS imaging of frozen hydrated HeLa cells. Surf Interface Anal 45: 302-304

Takáts Z, Wiseman JM, Gologan B, Cooks RG (2004) Mass spectrometry sampling under ambient conditions with desorption electrospray ionization. Science 306:471-473

Weismann D, Binder CJ (2012) The innate immune response to products of phospholipid peroxidation. Biochim Biophys Acta 1818:2465-2475 